of Science databases. With the assistance of a librarian, we used various combinations of keywords such as Moyamoya disease, ischemic stroke, hemorrhagic stroke, antiplatelet, and the names of individual antiplatelet agents. Titles and abstracts were screened by the first two authors (MAS and MEE), with a full-text review of relevant papers. Articles were included if they examined the effect of antiplatelet therapy on ischemic or hemorrhagic stroke or survival in patients with moyamoya disease.

Results Out of 132 retrieved studies, we identified five eligible studies published between 2014 and 2019 and included 26,605 patients (9499 on antiplatelet therapy, and 17106 controls) with follow up ranging from post-operative period to 6.3 years. Only one study had a prospective design and two studies were multi-center. Three of the studies included post-bypass patients, and one included only asymptomatic moyamoya disease. The antiplatelet therapy regimens varied across the studies and included ASA, clopidogrel, or cilostazol.Three studies had a control arm that did not receive any antiplatelet therapy. In these studies, the pooled risk for a composite outcome (any ischemic or hemorrhagic stroke, or death) was lower in the antiplatelet group compared to controls (9\% vs $11 \%$; relative risk 0.85 , 95\% CI: 0.79 to $0.92)$.

Conclusions The use of antiplatelet therapy in patients with moyamoya disease may confer better stroke-free survival. Multicenter prospective studies with a uniform antiplatelet protocol are needed to elucidate the potential benefits of this intervention.

Disclosures M. Suheel: None. M. Eagles: None. M. Almekhlafi: None.

\section{E-031 DURAL ARTERIOVENOUS FISTULAS WITHOUT CORTICAL VENOUS DRAINAGE: PRESENTATION, TREATMENT AND OUTCOMES}

${ }^{1} E$ Samaniego*, ${ }^{2} \mathrm{~J}$ Roa, ${ }^{3} \mathrm{M}$ Hayakawa, ${ }^{1} S$ Ortega-Gutierrez, ${ }^{4} \mathrm{R}$ Guniganti, ${ }^{5} \mathrm{D}$ Bulters, ${ }^{6} \mathrm{~A}$ Alaraj, ${ }^{6} \mathrm{~S}$ Amin-Hanjani, ${ }^{7} \mathrm{G}$ Lanzino, ${ }^{4} \mathrm{G}$ Zipfel, ${ }^{8} \mathrm{D}$ Hasan, ${ }^{3} \mathrm{C}$ Derdeyn. ${ }^{1}$ Neurology, Neurosurgery and Radiology, University of lowa Hospitals and Clinics, lowa City, IA; ${ }^{2}$ Neurology and Neurosurgery, University of lowa Hospitals and Clinics, lowa City, IA; ${ }^{3}$ Radiology, University of lowa Hospitals and Clinics, lowa City, IA; ${ }^{4}$ Neurosurgery, Washington University School of Medicine, St. Louis, MO; ${ }^{5}$ Neurosurgery, University Hospital Southampton, Southampton, UK; ${ }^{6}$ Neurosurgery, University of Illinois Hospital, Chicago, IL; ${ }^{7}$ Neurosurgery, Mayo Clinic, Rochester, MN; ${ }^{8}$ Neurosurgery, University of lowa Hospitals and Clinics, lowa City, IA

\subsection{6/neurintsurg-2020-SNIS.67}

Background Current evidence suggests that intracranial dural arteriovenous fistulas (dAVFs) lacking cortical venous drainage (CVD) have a benign clinical course. However, there is no large study evaluating the safety/efficacy of current treatments and their impact over the natural history of no-CVD dAVFs.

Methods We conducted an analysis of the retrospectively collected multi-center Consortium for dAVF Outcomes Research (CONDOR) database. Demographics, presenting symptoms, dAVFs' angiographic features and therapeutic intervention/complications data of patients with Borden-Shucart type 1 dAVFs were reviewed. Clinical and radiological follow-up information was assessed to determine rates of new intracranial hemorrhage or non-hemorrhagic neurological deficit (NHND), worsening of venous hyperdynamic symptoms (VHS), angiographic

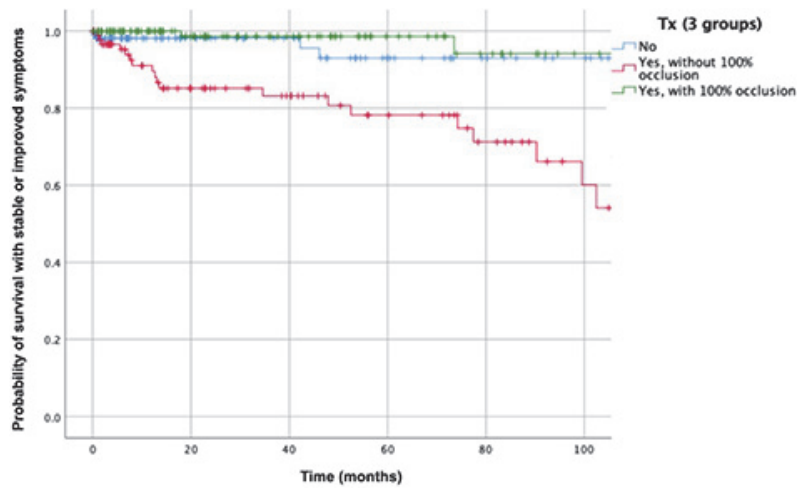

Abstract E-031 Figure 1 Subgroup Kaplan-Meier survival analysis for treated type I dAVFs that achieved complete angiographic obliteration

recurrence, progression or spontaneous regression of dAVFs over time.

Results A total of 368 patients/Borden-Shucart type I dAVFs were identified. For patients with multiple dAVFs, only the largest one was included in the analysis. Mean age was $57.9 \pm 15.6$ years, and $60.9 \%$ were women. Mean followup time was $40.1 \pm 45.2$ months. The most common location was the transverse/sigmoid sinus (50.3\%). Of 240 treated dAVFs, 224 (93.3\%) underwent endovascular embolization, $11(4.9 \%)$ radiosurgery alone and 5 (2.1\%) open surgery as primary modality. After first embolization, most dAVFs (45.5\%) achieved only partial reduction in early venous filling. Multiple complementary interventions increased complete obliteration rates from $37.5 \%$ after first embolization to $45.5 \%$ after 2 or more embolizations, and $53.8 \%$ after complimentary radiosurgery/open surgery. Immediate post-procedural complications occurred in 38 treated dAVFs (15.8\%) and 7 with permanent sequelae. Of 129 completely obliterated dAVFs by any therapeutic modality, $3(2.3 \%)$ showed angiographic recurrence/recanalization in a mean time of 10 months after treatment. Progression to Borden-Shucart types II-III was documented in $2.4 \%$ and subsequent development of new dAVF in $1.5 \%$. Partial spontaneous regression was found in 24 out of 115 non-treated dAVFs with follow-up available (20.9\%). Multivariate Cox regression analysis demonstrated that NHND or severe VHS at presentation and infratentorial location were associated with worse prognosis. Kaplan-Meier curves demonstrated no significant difference for stable/improved symptoms survival probability in treated versus non-treated dAVFs. However, estimated survival times showed better trends for treated dAVFs compared with non-treated dAVFs (179.2 months vs 163 months, Log-rank p-value $=0.12$ ). This difference was statistically significant for treated dAVFs with $100 \%$ occlusion compared with partiallyoccluded dAVFs (173.2 months vs 143.9 months, Log-rank p-value $<0.001)$.

Conclusion Current therapeutic modalities for management of dAVFs without CVD are safe and may provide better symptom control when complete angiographic occlusion can be achieved.

Disclosures E. Samaniego: 1; C; SVIN 2019, Bee Foundation 2019. 2; C; Medtronic, MicroVention. J. Roa: None. M. Hayakawa: None. S. Ortega-Gutierrez: 2; C; Stryker, Medtronic. R. Guniganti: None. D. Bulters: None. A. Alaraj: None. S. Amin-Hanjani: None. G. Lanzino: None. G. Zipfel: None. D. Hasan: None. C. Derdeyn: None. 